\title{
Distractor inhibition is more effective at a central than at a peripheral location
}

\author{
ZHE CHEN \\ University of Canterbury, Christchurch, New Zealand \\ AND \\ Anne Treisman \\ Princeton University, Princeton, New Jersey
}

\begin{abstract}
The "distractor eccentricity effect" refers to the finding of reduced interference from an incompatible distractor at a central relative to a peripheral location (Chen, 2008). The present study examines the mechanism that underlies the distractor eccentricity effect, and relates it to the inattentional blindness explored by Mack and Rock (1998), which was also more marked at a foveal than at a parafoveal location. The results suggest that these two visual phenomena may reflect the same underlying mechanism - a gradient of increasing attentional suppression from the periphery to the center.
\end{abstract}

It has long been established that the fovea plays an important role in the perception of color and fine details (Boynton, 1979; Rodieck, 1973). It has higher visual acuity, spatial resolution, and contrast sensitivity relative to the periphery (Connolly \& Van Essen, 1984; Fiorentini $\&$ Berardi, 1991), and it is also favored in the distribution of attention (Wolfe, O'Neill, \& Bennett, 1998). In the absence of special instructions to attend elsewhere, a stimulus at the fovea is processed more efficiently than the same stimulus at the periphery (Linnell \& Humphreys, 2004; Wolfe et al., 1998). This effect has been termed the eccentricity effect (Carrasco, Evert, Chang, \& Katz, 1995). When the relevant stimulus is at the fovea, this allocation of attention is clearly advantageous. However, if the foveal stimulus is irrelevant to the task, attending to it could be counterproductive, increasing the interference it generates with the task at hand. Ideally, one would like an attentional system that not only reduces distractor interference in general but also reduces it in such a way that the reduction is proportional to the potential conflict between the distractors and the target.

There is considerable evidence that active inhibition is an important component of selective attention (Dagenbach \& Carr, 1994). For example, probe detection is slower when the probe appears at a location previously occupied by a distractor rather than at a blank location between distractors (Cepeda, Cave, Bichot, \& Kim, 1998). Reaction time (RT) is also longer when a target was a distractor on a preceding trial than when it had not previously appeared (see Tipper, 2001, for a review; but also see Milliken, Joordens, Merikle, \& Seiffert, 1998; Neill, Valdes, Terry, \& Gorfein, 1992; and Park \& Kanwisher, 1994, for noninhibition interpretations). This negative priming effect has been found with different types of stimuli in a variety of paradigms (e.g., DeSchepper \& Treisman, 1996; Tipper \& Driver, 1988). Neurophysiological findings also suggest a role for inhibition in visual selection. When monkeys were required to make a saccade to either a "good" stimulus (one that elicits strong responses) or to a "poor" stimulus, responses of inferior temporal neurons to the "good" stimulus when it was not the target were suppressed before the onset of the saccade (Chelazzi, Miller, Duncan, \& Desimone, 1993). Attentional modulation has been observed in many parts of the brain (Chelazzi et al., 1993; Luck, Chelazzi, Hillyard, \& Desimone, 1997; Moran \& Desimone, 1985), suggesting that inhibition is widely used in visual perception.

The retinal distribution of distractor interference is still unclear. The discussion outlined above suggests two opposite predictions: Preferential attention to the fovea should make distractors there harder to ignore. On the other hand, this could promote stronger inhibition of distractors at the fovea to counteract their greater potential to disrupt performance, resulting in stronger interference from distractors in the periphery. The data so far also disagree. Whereas Beck and Lavie (2005) showed greater interference from an incongruent distractor at the fovea than from the same distractor in the periphery, other studies have found a peripheral distractor harder to ignore than a foveal one (Bouma, 1973; Chen, 2008; Goolkasian, 1999; Jonides, 1981; Juola, Koshino, \& Warner, 1995). In Beck and Lavie's experiment (Experiment 6), participants searched for a target embedded among several neutral distractors, with an additional critical distractor presented at either a foveal

Z. Chen, zhe.chen@canterbury.ac.nz 
or a peripheral location. The response evoked by the critical distractor could be either compatible or incompatible with the target response. A larger response compatibility effect (Eriksen \& Eriksen, 1974) was found when the distractor was at fixation than when it was in the periphery. Beck and Lavie attributed their result to the preferential access to attention by the fovea.

On the other hand, other researchers have reported greater interference from a peripheral distractor than from a central one. Both Jonides (1981) and Juola et al. (1995) found that a central cue is easier to ignore than a peripheral cue is. Goolkasian (1999) showed a spatial asymmetry in interference, with an incompatible peripheral distractor exerting more interference on a central target than the reverse. Chen (2008), holding all other factors constant, also demonstrated more interference from an incompatible distractor at a peripheral than at a central location. She suggested that the inconsistent results in prior studies, rather than reflecting an inherent feature of the visual system, could be explained by different response strategies. In Beck and Lavie's (2005) study, the distractor was compatible with the target on half the trials, encouraging participants to pay some attention to the distractor as well as to the target. If the fovea was not actively inhibited in their experiment, an incongruent foveal distractor would get more attentional resources and therefore cause greater interference than an incongruent peripheral distractor.

To test the effect of the type of distractors, Chen (2008) compared blocks in which half the distractors were compatible with blocks in which half were neutral. In both cases, the other half of them were incompatible. The assumptions were that distractor inhibition would be less likely to be evoked when there was a substantial number of compatible distractor trials, and that this would result in stronger interference from incompatible distractors at the fovea than at the periphery (as found by Beck \& Lavie, 2005). As predicted, when half the distractors were compatible, interference on incompatible trials was stronger with the distractor at the fovea than at the periphery. However, when only incompatible or neutral distractors were presented, this effect disappeared. Furthermore, when the allocation of attention was carefully controlled in subsequent experiments, participants showed the distractor eccentricity effect: stronger interference from peripheral than from central distractors on incompatible trials. Participants presumably inhibited the fovea to minimize distractor interference and as a result showed more interference from the peripheral distractor.

Mack and Rock (1998) described a phenomenon that may be related. When participants were focused on another task, they often failed to detect an unexpected stimulus, even though it would otherwise have been well above threshold. Interestingly, this inattentional blindness (IB) was more marked when the unexpected stimulus was at fixation rather than at a parafoveal location.

The present experiments investigated whether the mechanism that gives rise to the distractor eccentricity effect is inhibitory in nature. Experiments 1 and 2 also examined the relationship between the distractor eccentricity effect and Mack and Rock's (1998) finding of stronger IB at a foveal than at a parafoveal location. An alternative account of the distractor eccentricity effect might be that the speed of processing is faster in the periphery than at the fovea (Carrasco, Giordano, \& McElree, 2006; Carrasco, McElree, Denisova, \& Giordano, 2003), and that this results in earlier and therefore greater interference from peripheral than from foveal distractors. Experiments $3 \mathrm{~A}$ and $3 \mathrm{~B}$ tested this possibility in our paradigm, and confirmed that the processing speed of the central and peripheral stimuli used in the present paradigm was comparable. Experiment 4 further investigated the mechanism that underlies the distractor eccentricity effect by using a spatial probe technique (Kim \& Cave, 1995) that measured the allocation of spatial attention at the location of the critical distractor at $1^{\circ}$ or $9^{\circ}$ eccentricity. Together, these experiments explore the flexibility of the visual attention system - in particular, the idea that it can apply different levels of inhibition to counterbalance different degrees of interference that would otherwise occur at different retinal locations.

\section{EXPERIMENT 1}

The goal of Experiment 1 was to assess both distractor interference at different eccentricities and IB in the same experiment. Experiment 1 included two types of trials: Most were letter discrimination trials, in which participants made a speeded response to a target embedded in an array that also contained two identical distractors. An additional critical distractor was presented at either a central or a peripheral location, and it could be incompatible (the other target letter), neutral (a letter not associated with the required responses), or, on a small proportion of trials, compatible (the same letter as the target).

In addition, at the end of the experiment there were two unexpected probe recognition trials, in which the critical distractor was replaced by a rectangle. The task was to identify the unexpected rectangle in addition to responding to the target letter. The two main questions were (1) whether the distractor eccentricity effect would be observed in the letter discrimination task; and (2) whether stronger IB would be found when the unexpected probe shape was at a central rather than a peripheral location.

\section{Method}

Participants. Twenty-four Princeton University undergraduate students participated in the experiment in exchange for course credit. All of them reported having normal or corrected-to-normal vision.

Apparatus and Stimuli. All stimuli were light gray, presented against a black background. They were shown on a PC with a 22-in. monitor, and E-Prime (Schneider, Eschman, \& Zuccolotto, 2002) was used to generate stimuli and collect responses.

Each letter discrimination trial consisted of a fixation display, a cue, and a target display (see Figure 1). The fixation display consisted of a small dot at the center and two identical pairs of vertically aligned bars, one on each side of the screen, with the center of each pair located at $5^{\circ}$ eccentricity left or right of fixation. The cue consisted of one pair of bars identical to those in the fixation display, and it was equally likely to be on the left or right side of the screen. We used the cue to ensure that the participants' attention was directed to the target location rather than to the center, since there is evidence that the default mode of attention distribution is to favor central stimuli over peripheral ones (Chen, 2008; Wolfe et al., 1998). The target display, which always appeared on the same side of the screen as the 


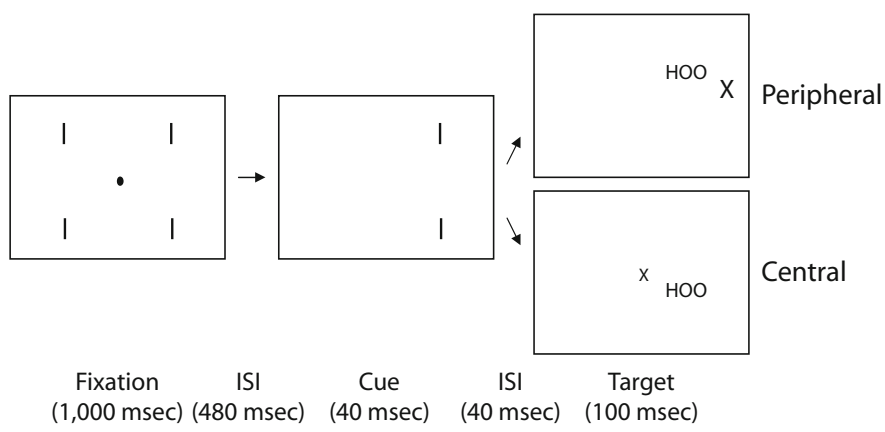

Figure 1. Examples of stimulus displays from Experiment 1. The task was to search for an $\mathrm{H}$ or an $\mathrm{X}$ flanked by two identical letter Os. The critical distractor, whose relationship with the target was manipulated, was equally likely to appear on the left or right side of fixation at a central location of $1^{\circ}$ eccentricity or at a peripheral location of $9^{\circ}$ eccentricity.

cue, contained a three-letter target array (the target $\mathrm{H}$ or $\mathrm{X}$ with two neutral letter Os) and a critical distractor $(\mathrm{H}, \mathrm{X}$, or L). The three letters in the target array, each of which subtended $0.86^{\circ}$ in height, were horizontally aligned, with their centers located $7.16^{\circ}, 5.25^{\circ}$, and $3.34^{\circ}$ from the fixation. They were equally likely to be above or below the horizontal meridian, with the center of the middle letter vertically aligned with the cue. The target could appear at any of the three positions in the target array with equal probability. The critical distractor always appeared on the horizontal meridian, and was equally likely to be at $1^{\circ}$ eccentricity (the central condition) and at $9^{\circ}$ eccentricity (the peripheral condition). The size of the distractor was scaled relative to that of the middle letter of the target array in accordance with the cortical magnification factor (Rovamo \& Virsu, 1979; Virsu \& Rovamo, 1979). It extended $0.48^{\circ}$ at $1^{\circ}$ eccentricity (size 9 and bold), and $1.38^{\circ}$ at $9^{\circ}$ eccentricity (size 24 and plain). The participants viewed the display from a distance of $60 \mathrm{~cm}$.

Design and Procedure. The sequence of displays is shown in Figure 1 . The task was to make a speeded response to the identity of the target by pressing one of two labeled keys: the "." key if the target was an $\mathrm{H}$, and the "/" key if it was an X. The principal manipulations were distractor eccentricity $\left(1^{\circ}\right.$ or $\left.9^{\circ}\right)$ and response incompatibility between the target and the critical distractor (neutral or incompatible). Both factors were manipulated within a block. A compatible distractor was also used on one ninth of the trials. These were filler trials whose function was to discourage participants from guessing the target response via the identity of the critical distractor. ${ }^{1}$ On the remaining trials, the critical distractor was equally likely to be neutral or incompatible.

There were two unexpected probe recognition trials. They occurred on the last and the fourth-from-last trial. On these trials, the critical distractor was replaced by an outline rectangle of the same size. The rectangle was at a central location for half of the participants, and at a peripheral location for the other half. The participants still responded to the target letter. However, upon response, they were immediately asked whether there was anything unusual in the previous display. Regardless of their answer, they were shown four shapes (a rectangle, a triangle, a circle, and a diamond on the same horizontal row and in that order), and were required to select the one that appeared on the previous trial, guessing if they had to. Following Mack and Rock (1998), we refer to the first unexpected trial as the surprise trial, and the second one as the divided attention trial. The experiment consisted of 650 letter discrimination and two unexpected probe recognition trials. It took approximately $45 \mathrm{~min}$ to complete.

\section{Results and Discussion}

The results for the regular trials are shown in Figures $2 \mathrm{~A}$ and $2 \mathrm{~B}$. A repeated measures ANOVA indicated faster RTs on neutral trials $(550 \mathrm{msec})$ than on incompatible trials $(584 \mathrm{msec})\left[F(1,23)=80.77, M S_{\mathrm{e}}=345.8, p<\right.$ $.001]$, and more importantly, more interference from the incompatible distractor at the peripheral $(46 \mathrm{msec})$ than at the central $(23 \mathrm{msec})$ location $\left[F(1,23)=6.69, M S_{\mathrm{e}}=\right.$ $301.0, p<.05]$. A similar analysis on the accuracy data indicated a significant incompatibility effect $[F(1,23)=$ $\left.14.20, M S_{\mathrm{e}}=8.5, p<.01\right]$, with lower accuracy on incompatible trials $(6.3 \%$ error) than on neutral ones $(4.1 \%$ error). No other effects reached significance, and there was no evidence of a speed-accuracy trade-off.

The data for the unexpected probe trials are in Figure $2 \mathrm{C}$. On the divided attention trial, the probability of detecting the probe was significantly higher in the peripheral condition than in the central condition $\left[\chi^{2}(1)=4.44\right.$, $p<.05]$. Unfortunately, the result did not reach significance on the surprise trial $\left[\chi^{2}(1)=2.27, p=.13\right]$, even though there were more detections with the peripheral probe. This null result is likely due to a floor effect, since the overall probe recognition rate was not significantly different from guessing on the surprise trial.

Although the results of Experiment 1 were consistent with the notion that the distractor eccentricity effect and IB may reflect the same underlying mechanism, the fact that we did not obtain a statistically significant difference between the central and peripheral conditions on the surprise trial prevents us from drawing an unequivocal conclusion. Experiment 2 was designed to increase the sensitivity of the experiment by improving the overall performance on the surprise trial.

\section{EXPERIMENT 2}

In Experiment 1, the critical stimulus on the unexpected probe recognition trials was an outline rectangle. In Experiment 2, we replaced that with a schematic happy face. Mack and Rock (1998) reported a reduction in IB when the critical stimulus was a schematic smiling face relative to a scrambled or a sad face, suggesting that a smiling face may be less susceptible to IB. There is considerable evidence that faces are special stimuli whose processing differs from that of common objects (Bruce, 1988; Purcell 

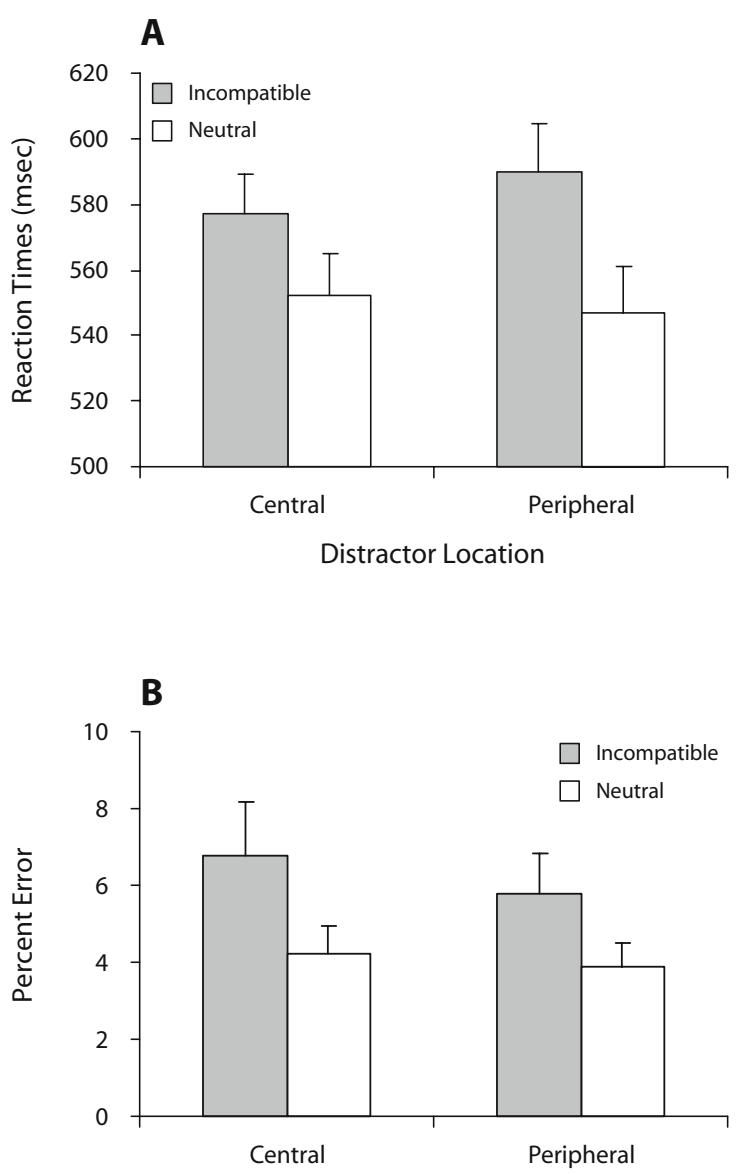

Distractor Location

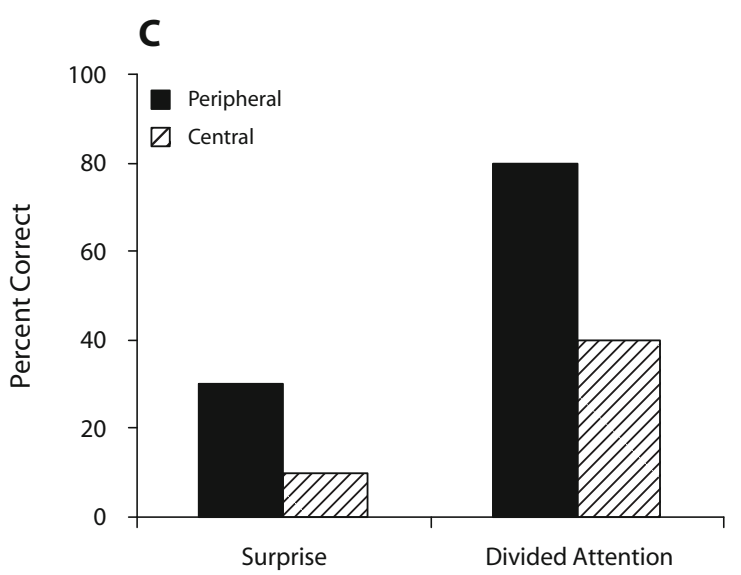

Type of Trial

Figure 2. Results of Experiment 1. (A) Reaction times as a function of distractor location and response incompatibility in the letter discrimination task. (B) Percent error for the letter discrimination task. (C) Percent correct for the unexpected probe recognition trials.
\& Stewart, 1986). Face-selective neurons have also been found in the superior temporal sulcus of macaques (Desimone, 1991; Gross, Rocha-Maranda, \& Bender, 1972). Furthermore, fMRI has shown the fusiform gyrus in normal healthy people to be more active when they view faces relative to other objects such as houses, scrambled faces, or animals (Kanwisher, McDermott, \& Chun, 1997; but see Gauthier, Skudlarski, Gore, \& Anderson, 2000). These findings suggest that there may be a special perceptual mechanism for detecting faces, which could increase the probability of detecting a schematic smiling face on the critical trial, even though attention was directed elsewhere.

\section{Method}

The method was the same as that of Experiment 1, except for the following two differences. First, the probe on the unexpected recognition trial was replaced by a schematic happy face. Consequently, the stimuli in the probe choice display were changed to a triangle, a happy face, a circle, and an unhappy face. Second, the compatible condition was excluded from the experiment. The experiment consisted of 386 letter discrimination trials, in addition to two unexpected probe recognition trials. Twenty-six participants from the University of Canterbury took part in the experiment.

\section{Results and Discussion}

Figures $3 \mathrm{~A}$ and $3 \mathrm{~B}$ show the results for the letter discrimination task. Participants were faster and more accurate on neutral (586 msec with $4.5 \%$ error) than on incompatible $(620.5 \mathrm{msec}$ with $8.5 \%$ error) trials $[F(1,25)=$ $104.5, M S_{\mathrm{e}}=308.1, p<.001$ for RT, and $F(1,25)=26.6$, $M S_{\mathrm{e}}=15.4, p<.001$ for accuracy]. Furthermore, the response incompatibility effect was larger when the incompatible distractor was at the peripheral $(46 \mathrm{msec}$ with $5.5 \%$ error) than at the central ( $24 \mathrm{msec}$ with $2.9 \%$ error) location $\left[F(1,25)=6.85, M S_{\mathrm{e}}=352.7, p<.05\right.$, and $F(1,25)=6.53, M S_{\mathrm{e}}=8.23, p<.05$, for RT and accuracy, respectively]. There was no significant main effect of critical distractor location in either RT or accuracy.

Figure $3 \mathrm{C}$ shows the data for the unexpected probe trials. On the surprise trial, IB was stronger in the central condition than in the peripheral condition $\left[\chi^{2}(1)=3.94\right.$, $p<.05]$. Further analyses revealed that, whereas the recognition performance was significantly better than chance when the unexpected probe was at the peripheral location $\left[\chi^{2}(1)=9.25, p<.05\right]$, it was at chance when the probe was at the central location $\left[\chi^{2}(1)=0.025, p=.87\right]$. On the divided attention trial, there was no difference in IB between the central and peripheral conditions $\left[\chi^{2}(1)=\right.$ $0.87, p=.35]$. It is likely that the overall increase in the probe recognition rate eliminated this difference.

The most important finding of Experiment 2 is that both the distractor eccentricity effect and the IB results reported by Mack and Rock (1998) were replicated in the same paradigm. By using a happy face as the critical unexpected stimulus, we were able to eliminate the floor effect on the surprise trial, at least in the peripheral condition.

Mack and Rock (1998) attributed strong IB at fixation to active suppression of foveal stimuli. They reasoned that, because attention is normally paid to objects at the fovea, when a target is not expected there, active inhibition is 

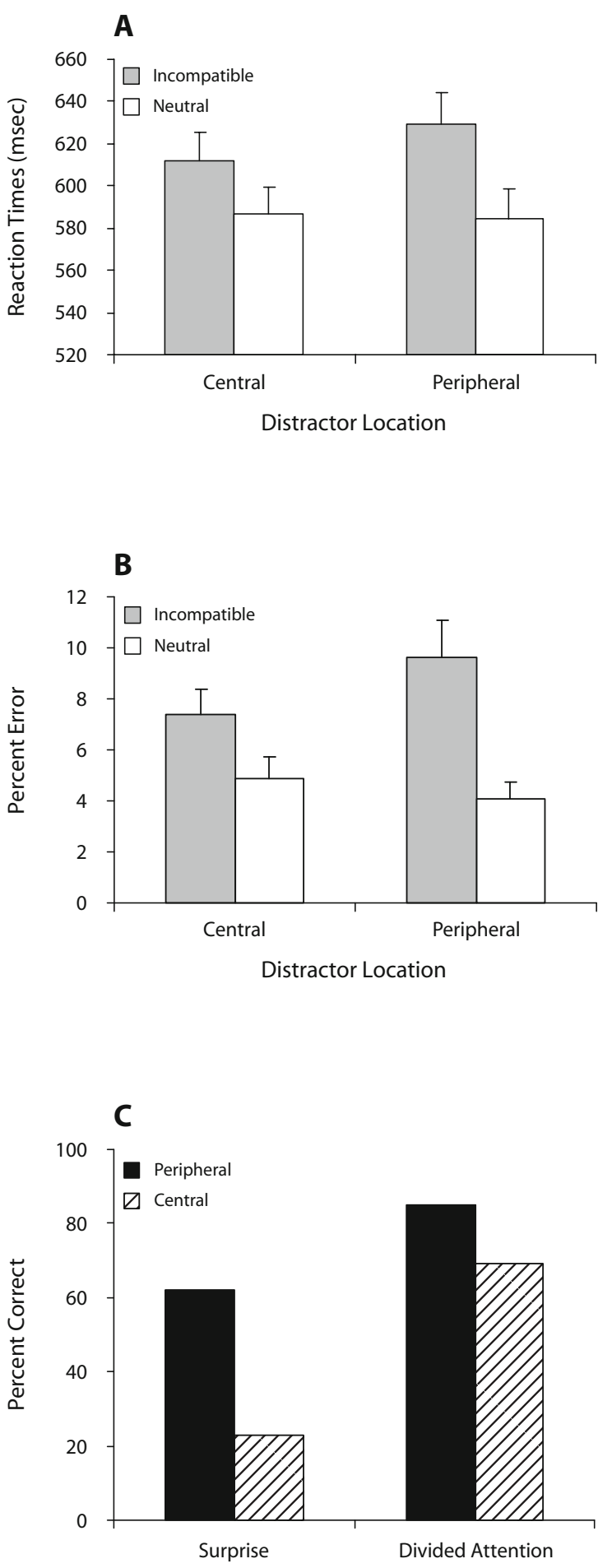

Type of Trial

Figure 3. Results of Experiment 2. (A) Reaction times as a function of distractor location and response incompatibility in the letter discrimination task. (B) Percent error for the letter discrimination task. (C) Percent correct for the unexpected probe recognition trials. evoked to suppress the fovea, resulting in strong IB when an unexpected stimulus occurs there. Unfortunately, as Beck and Lavie (2005) pointed out, Mack and Rock's result could be influenced by the target having more location uncertainty and a larger eccentricity when the unexpected probe was at the fovea rather than at a parafoveal location. In our experiment, both these factors were controlled, yet we still replicated the differential IB reported by Mack and Rock. The fact that the distractor eccentricity effect and differential IB were observed in the same paradigm suggests that the two phenomena may reflect the same underlying mechanism.

\section{EXPERIMENTS 3A AND 3B}

In the next several experiments, we explored an alternative interpretation. Carrasco and her associates recently reported that the speed of visual information processing increases with eccentricity (Carrasco et al., 2006; Carrasco et al., 2003). In a series of experiments, they used a response signal speed-accuracy trade-off procedure (Reed, 1973; Wickelgren, 1977). The participants were required to respond within $300 \mathrm{msec}$ of a tone. The results showed that orientation discrimination was faster for a target at $9^{\circ}$ eccentricity than at $4^{\circ}$ eccentricity. Furthermore, this peripheral advantage was observed whether the target was presented alone or with irrelevant distractors; whether the stimulus size was scaled or constant; and whether the location of the target was precued or not precued. If the peripheral distractor in our experiment was processed faster than the more central one, it would have more time to interfere, and this could explain our distractor eccentricity effect.

In order to investigate whether this alternative account should replace our hypothesis that the reduced interference from the central distractor was due to greater inhibition of distractors close to the fovea, the next two experiments tested directly the relative speed of processing at the center and at the periphery in our displays in conditions in which no inhibition should be invoked.

In Experiments 1 and 2, we scaled the size of the critical distractor in accordance with the cortical magnification factor (Rovamo \& Virsu, 1979; Virsu \& Rovamo, 1979), assuming that the visual acuity of the distractor at $1^{\circ}$ vs. $9^{\circ}$ eccentricity would thereby be equated. In Experiments $3 \mathrm{~A}$ and $3 \mathrm{~B}$, we verified this assumption empirically. The task was to respond to a target letter $\mathrm{H}$ or $\mathrm{X}$ at $1^{\circ}$ or $9^{\circ} \mathrm{ec}-$ centricity. The target was presented either alone (Experiment 3A) or with distractors (Experiment 3B). Of specific interest was whether participants would show comparable RTs and accuracy for stimuli at these two eccentricities.

\section{Experiment 3A}

\section{Method}

The stimuli were identical to those in Experiment 1, except that the target display consisted of a single letter $\mathrm{H}$ or $\mathrm{X}$ at $1^{\circ}$ or $9^{\circ}$ eccentricity. The target had the same sizes and styles as those of the critical distractor in Experiment 1. Eleven new participants from Princeton University took part in the experiment. They completed 180 trials in two blocks. 


\section{Results}

There was no significant effect of central versus peripheral location of the target $(418 \mathrm{msec}$ with $6.3 \%$ error for the central location and $422 \mathrm{msec}$ with $7.3 \%$ error for the peripheral location) $[t(10)=0.85, p=.42$, and $t(10)=0.69, p=$ .51 , for RT and accuracy, respectively]. (See Experiment 3B for the discussion of the results of this experiment.)

\section{Experiment 3B}

In case the speed of processing at different locations was affected by the presence of distractors, Experiment $3 \mathrm{~B}$ replicated Experiment $3 \mathrm{~A}$ with the addition of three distractor letters.

\section{Method}

The target display again consisted of a target letter $\mathrm{H}$ or $\mathrm{X}$ at $1^{\circ}$ or $9^{\circ}$ eccentricity (as that of Experiment $3 \mathrm{~A}$ ). A three-letter distractor array was added, its size and location identical to those of the three-letter target array in Experiment 1 . The only difference between these two arrays was that in Experiment 3B the target letter, $\mathrm{H}$ or $\mathrm{X}$, was presented in the critical distractor locations of Experiment 1 , and the letter $\mathrm{L}$ replaced the $\mathrm{H}$ or $\mathrm{X}$ in the three-letter array of Experiment 1. Eleven new participants from the University of Canterbury took part in the experiment. Each completed 180 trials divided into two blocks.

\section{Results and Discussion}

As in Experiment 3A, no significant difference was found in RT or accuracy between the targets in the central condition (529 msec with $5.9 \%$ error) and those in the peripheral condition $(540 \mathrm{msec}$ with $5.2 \%$ error) $[t(10)=$ $1.29, p=.22$ for $\mathrm{RT}$, and $t(10)=0.81, p=.44$ for accuracy]. These results are consistent with the predictions of Rovamo and Virsu (1979; Virsu \& Rovamo, 1979). They provide empirical support for the assumption that the critical distractors of Experiments 1 and 2 at the central and peripheral locations were matched in discriminability and speed of processing.

Carrasco et al.'s (2006; Carrasco et al., 2003) experiments tested the eccentricity effect on speed of processing in conditions where participants were required to respond within $300 \mathrm{msec}$ of a tone. To check whether our results in Experiments $3 \mathrm{~A}$ and $3 \mathrm{~B}$ exhibited a similar pattern of data on fast trials, we divided the raw RT data into two equal categories, with the fast half into the fast category and the slow half into the slow category. We found no significant differences in RTs between the central and peripheral conditions in either category. ${ }^{2}$

Because of the many differences in methodology between Carrasco et al.'s (2006; Carrasco et al., 2003) experiments and the present experiments, our result does not contradict their claim. It simply rules out differences in the speed of processing as an explanation for our findings of the distractor eccentricity differences in Experiments 1 and 2 . Eccentricity differences may result from a variety of different factors in these experiments, including the absence or presence of a response deadline, the locations of the central target $\left(1^{\circ} \mathrm{Vs} .4^{\circ}\right)$ and the precue (at a nontarget location vs. a target location), the type of stimuli (letters vs. Gabor patches), and the task requirement (letter vs. orientation discrimination). Regardless of what causes the differences, the important point about the present study is that the distractor eccentricity effect observed in our experiments was not caused by differential processing speed of a central and a peripheral distractor.

\section{EXPERIMENT 4}

In Experiment 4, we carried out a direct test of the distractor inhibition account, using a spatial probe technique (Kim \& Cave, 1995) to provide converging evidence for the inhibition account of the distractor eccentricity effect. In a typical spatial probe paradigm, participants perform a visual search task, but withhold their response to the target until a prompt appears. During the delay period, a probe sometimes appears at one of several locations and the participants make a speeded response to the probe. It has been shown that the probe detection time varies systematically with the type of stimuli that had occupied the location of the probe on a preceding display, presumably reflecting the activation or the inhibition that those stimuli had evoked in the locations they occupied. For example, RT to the probe is shorter when the probe appears at a location previously occupied by a target rather than by a distractor, and when the distractor was dissimilar rather than when it was similar to the target (Cave \& Zimmerman, 1997). These results suggest that RT to the probe is sensitive not only to the allocation of spatial attention but also to the magnitude of distractor inhibition.

Two groups of participants took part in the study. As in Experiment 1, they searched for an $\mathrm{H}$ or an X. However, instead of responding to the target immediately, they withheld their response until a prompt came on the screen. We measured accuracy rather than RT in this letter task. For half the participants (the distractor group), the target display was identical to that of Experiment 1. For the remaining participants (the no-distractor group), the critical distractor was not included in the target display. After the target display, on a small proportion of trials, a probe appeared at the location of the critical distractor, and participants made a speeded response to the probe. The probe appeared at the same locations for all participants, regardless of which group they belonged to. If the distractor eccentricity effect was caused by more efficient inhibition of the distractor at a central location than at a peripheral location, we should observe slower response latencies for a central probe than for a peripheral probe in the distractor group, but not in the no-distractor group, because the absence of the critical distractor in the latter group would render the inhibition of the distractor location unnecessary.

\section{Method}

The procedure was identical to that of Experiment 1, except for the following changes (see Figure 4). Sixteen new participants were equally divided and randomly assigned to the distractor and no-distractor groups. For both groups, $69 \%$ of trials were noprobe trials and $31 \%$ were probe trials. For the distractor group, the target display included a critical distractor (four ninths each for neutral and incompatible trials, and one ninth for compatible trials, just as in Experiment 1), but for the no-distractor group, the critical distractor was omitted. On no-probe trials, after a delay of $1,040 \mathrm{msec}$, a letter prompt with the phrase "H or X?" ap- 
No-Probe Trial
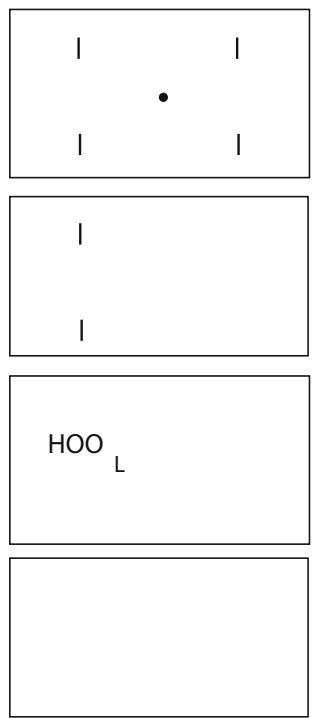

$\mathrm{H}$ or $\mathrm{X}$ ?
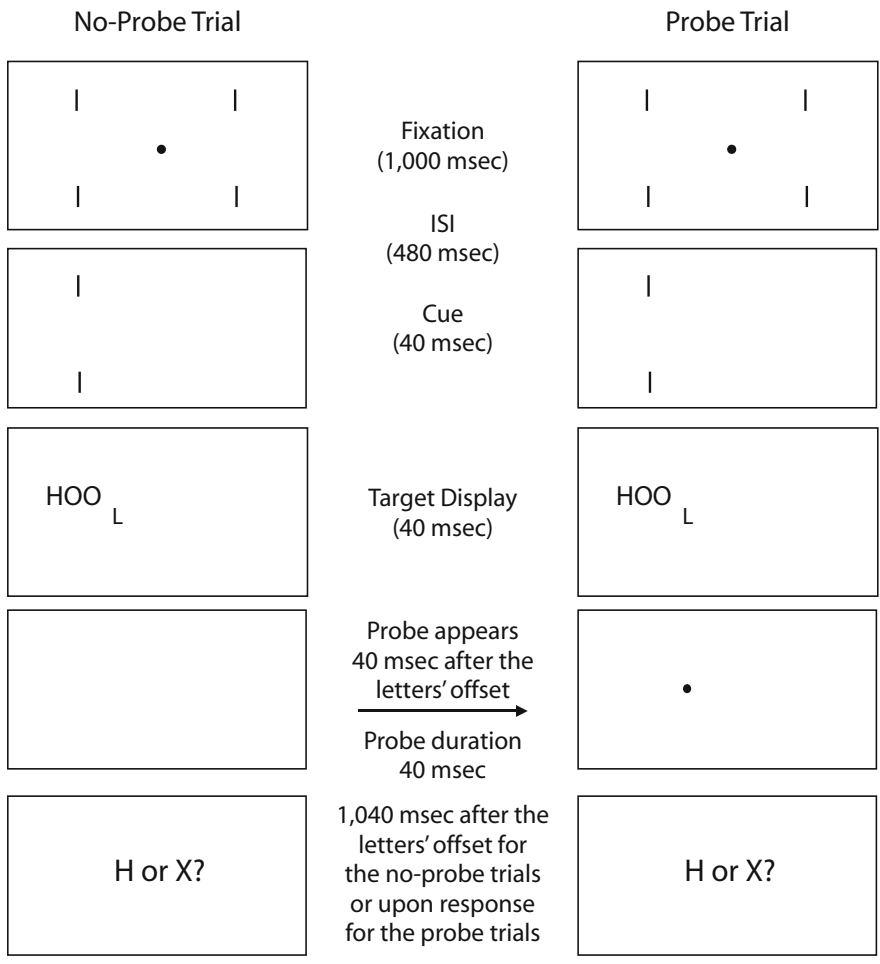

Figure 4. Examples of stimulus displays for the distractor group of Experiment 4. Participants withheld their responses to the target letter until a prompt appeared at the end of a trial. On about a third of trials (the probe trials), upon the offset of the target display, a small probe appeared at the location of the critical distractor, either at $1^{\circ}$ or $9^{\circ}$ eccentricity. Participants were required to respond to the probe as quickly as possible on those trials.

peared and remained on the screen until participants pressed a key to identify the target letter. On probe trials, $40 \mathrm{msec}$ after the target display, a probe (a small white dot subtending $0.19^{\circ}$ at $1^{\circ}$ eccentricity, or $0.43^{\circ}$ at $9^{\circ}$ eccentricity) appeared for $40 \mathrm{msec}$ in the location the distractor had occupied for the distractor group. The probe was equally often peripheral and central, and the distractor was always neutral. ${ }^{3}$ For the no-distractor group, the probe appeared in the same location, which was previously empty. To indicate the presence of the probe, participants pressed the " $Z$ " key with their left hands. The response to the probe triggered the presentation of the letter prompt, and participants pressed a key to identify the target letter. Speed was stressed for the probe task, and accuracy for the letter task.

\section{Results and Discussion}

The probe RT data are shown in Figure 5A. RT was faster for the no-distractor group $(412 \mathrm{msec})$ than for the distractor group $(549 \mathrm{msec})\left[F(1,14)=9.23, M S_{\mathrm{e}}=\right.$ $16,173.6, p<.01]$, and it was faster overall when the probe was at a peripheral location $(462 \mathrm{msec})$ than at a central location $(500 \mathrm{msec})\left[F(1,14)=8.13, M S_{\mathrm{e}}=1,393.0\right.$, $p<.05]$. The location $\times$ group interaction was also significant $\left[F(1,14)=5.79, M S_{\mathrm{e}}=1,393.0, p<.05\right]$, with faster RT in the peripheral condition $(514 \mathrm{msec})$ than in the central condition $(584 \mathrm{msec})[t(7)=2.68, p<.05]$ for the distractor group, but no significant difference between the conditions for the no-distractor group (409 msec vs.
$415 \mathrm{msec}$ for the peripheral and central conditions, respectively) $[t(7)=1.21, p=.27]$.

For the letter task, accuracy was high on the no-probe trials, and the two groups did not differ significantly $[t(14)=$ $0.57, p=.58] .{ }^{4}$ The mean error rates were $2.2 \%$ and $1.8 \%$ for the distractor and no-distractor groups, respectively. Accuracy was lower on the probe trials, with $10.6 \%$ error for the distractor group and 7.6\% error for the no-distractor group (see Figure 5B). The difference between the groups was not significant $[t(14)=0.87, p=.40]$. Interestingly, on the probe trials, the distractor group made more letter discrimination errors when the critical distractor was at a peripheral location $(14.5 \%$ error) than when it was at a central location $(6.8 \%)[t(7)=2.42, p<.05]$. This result suggests that the peripheral probe interfered with the processing of the target more than the central probe.

The finding that the probe RT was faster for the nodistractor group than for the distractor group is not surprising. ${ }^{5}$ This result could be caused either by the forward masking effect of the critical distractor, which appeared at the same location as the probe in the distractor group, or by the attentional interference caused by the distractor, or by both. It is worth noting, however, that the masking effect per se is unlikely to affect the probe detection times at the central and peripheral locations differentially, because 


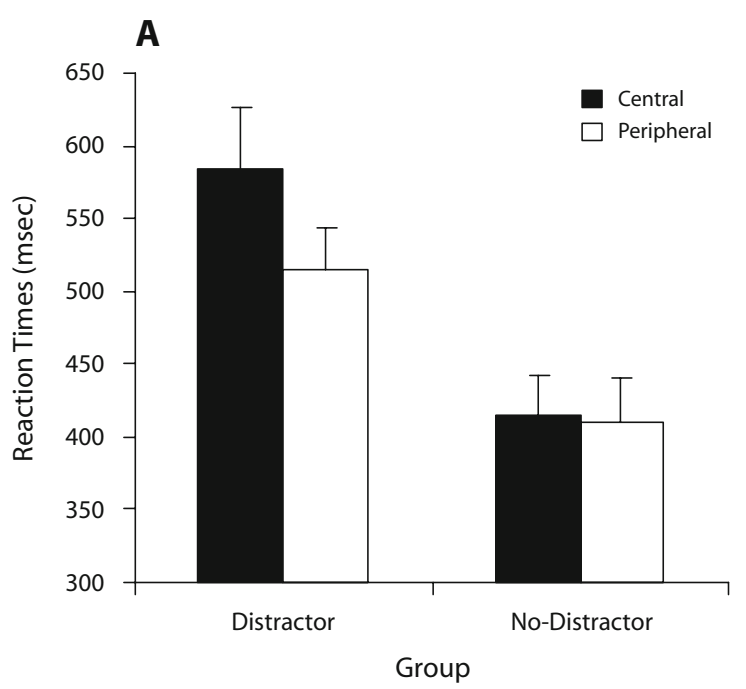

B

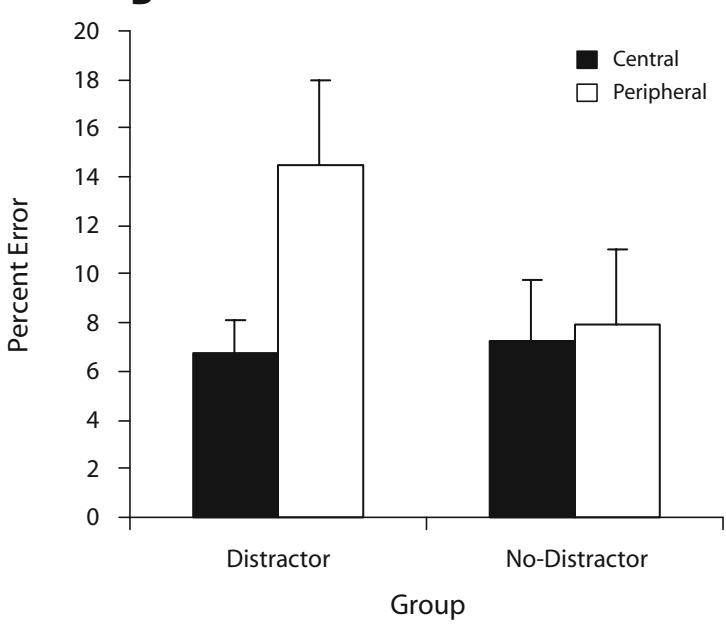

Figure 5. Results of Experiment 4. (A) Mean reaction times for the probe detection trials as anction of group and probe location. (B) Percent error for the letter discrimination task on the probe trials as a function of group and distractor location.

the sizes of both the critical distractor and the probe were scaled. In other words, whereas the masking effect could explain the main effect of group, it could not explain the group $\times$ location interaction.

As a precaution, we verified the above reasoning empirically. We conducted an additional dot detection experiment (see Appendix A). Participants $(n=8)$ saw stimulus displays that consisted of a fixation, a cue (a pair of vertically aligned bars) at $9^{\circ}$ or $1^{\circ}$ eccentricity on the left or right side of the screen, followed by either a letter $(\mathrm{H}$ or $\mathrm{X})$ at the cued location (the letter condition) or a blank screen (the no-letter condition), which in turn was followed by a target dot on eight ninths of the trials, or no dot on the remaining catch trials. As in Experiment 4, the dot always appeared at $9^{\circ}$ or $1^{\circ}$ eccentricity, whether or not it was preceded by a letter. Both the dot and the letter were scaled. The dot was identical to the probe target in Experiment 4, and the letter was the same as the critical distractor. The task was to press a designated key when a dot was present and to press the space bar to initiate the next trial when no dot appeared in the display.

The results are shown in Appendix B. The most important finding is that there was neither a main effect of condition nor a condition $\times$ location interaction. ${ }^{6}$ In other words, the probe detection times did not differ in the letter and no-letter conditions (365 msec vs. $369 \mathrm{msec}$ for the letter and no-letter conditions, respectively). Furthermore, the difference in RT between the central and peripheral locations was comparable in both conditions (35 for the letter condition and 32 for the no-letter condition). These results suggest that the differential probe detection times in the distractor and no-distractor groups of Experiment 4 were unlikely to be caused by forward masking.

The null result in the probe detection RTs for the nodistractor group of Experiment 4 also indicates that the distractor eccentricity effect observed in Experiments 1 and 2 and the differential probe detection times between the central and peripheral conditions for the distractor group of Experiment 4 were unlikely to have been caused by some sort of attentional momentum in the direction of the peripheral distractor. In both cases, participants shifted their attention away from the fovea on each trial. One could argue that the processing of the distractor and/ or probe at a peripheral location was facilitated by the launching of attention toward the peripheral location. However, the fact that no difference in the probe detection times between the central and peripheral conditions was observed for the no-distractor group makes the attentional momentum explanation unlikely.

In short, the results of Experiment 4 are consistent with the notion that the distractor eccentricity effect is caused by stronger inhibition for a central distractor than for a peripheral distractor. According to our hypothesis, the greater foveal interference results from the attentional deployment of inhibition to counteract the greater interference that would otherwise arise from foveal distractors.

\section{GENERAL DISCUSSION}

Prior research has established that an incompatible distractor causes more interference in the periphery than at the fovea (Chen, 2008; Goolkasian, 1999), and that an unexpected parafoveal stimulus is more likely to be detected than a foveal one (Mack \& Rock, 1998). In the present study, we replicated these findings within a single experimental paradigm. Moreover, we showed that participants were slower to respond to a probe at a central than at a peripheral location when the probe location was previously occupied by a distractor, but not when it was previously empty. These results suggest a common underlying mechanism for the distractor eccentricity effect and for the stronger foveal IB - namely, an increasing gradient of attentional suppression of distractors from the periphery 
to the center, mirroring the increasing gradient of attentional facilitation for relevant stimuli. The negative relationship between suppression and eccentricity may be a way for the visual system to counteract the higher acuity and salience of foveal stimuli when they are irrelevant to the task. Given that a task-irrelevant foveal distractor is likely to cause more interference than a peripheral one, it seems reasonable for the attention system to apply stronger suppression at the fovea than at the periphery.

Our results tested only two locations and therefore cannot on their own provide unequivocal support to the presence of a continuous gradient of attentional suppression from the periphery to the center. However, the idea of a gradient distribution of attention is not new. Several researchers (e.g., Downing \& Pinker, 1985; Shulman, Sheehy, \& Wilson, 1986) have shown that the cost of switching attention increases with an increase in the retinal separation between two locations; and that when the distance between the locations is held constant, the cost is larger for stimuli near the fovea than farther away. Bennett and Pratt (2001) showed that the magnitude of inhibition of return (Posner \& Cohen, 1984) decreased with distance from the cue. Jiang and Chun (2001) reported larger object substitution masking when the mask flanked the target on the peripheral side rather than on the central side. They proposed that this asymmetry was caused by stronger inhibition to the central side of the target than to the peripheral side. Dori and Henik (2006) further demonstrated that when attention is switched between two locations along an imaginary semicircle, RTs to a target on an outer semicircle are shorter than RTs to a target on an inner semicircle. Dori and Henik interpreted their result in terms of an inhibitory attentional gradient that centers around fixation, although they did not rule out the possibility that peripheral processing is faster. The present experiments distinguish a gradient of stronger distractor suppression from a gradient of faster processing from periphery to fovea, and support the notion of a gradient of attentional suppression that is the inverse of the gradient of attentional facilitation found earlier.

These spatial gradients of attentional inhibition suggest that attention operates within retinotopic visual areas. Indeed, recent research has found evidence for attentional suppression in striate and extrastriate visual areas (Smith, Singh, \& Greenlee, 2000; Somers, Dale, Seiffert, \& Tootell, 1999). For example, Smith et al. reported that when participants were attending to a target, they showed an increase in fMRI blood oxygenation level dependent (BOLD) signal activation at the location of the target. More importantly, the increase in BOLD activation at the attended location was accompanied by a decrease in activation at other locations from V1 to V4. These results are consistent with the view that visual attention is a multilevel selection process (see Kastner \& Pinsk, 2004, for a review). According to Kastner and Pinsk, selection starts in the lateral geniculate nucleus by enhancing neural responses to the attended stimulus relative to the unattended stimulus. Task irrelevant information is filtered out in in- termediate cortical areas such as V4 and TEO. Information from these extrastriate cortices is then integrated in higher order areas of the frontal and parietal cortex, which in turn provide top-down control via feedback connections to lower order areas in the visual system, either directly or through the pulvinar. Our results provide evidence that attention works, at least in part, by inhibiting locations where irrelevant competing stimuli appear. The inhibition is stronger around the fovea than in the periphery, consistent with our claim that distractors would otherwise produce more interference at the fovea.

\section{AUTHOR NOTE}

This research was supported in part by NIH Grant 2004 2RO1 MH 058383-04A1 (Visual Coding and the Deployment of Attention); by Grant 1000274 from the Israeli Binational Science Foundation; and by NIH Grant 1RO1MH062331 (Spatial Representations and Attention) to A.T. We thank two anonymous reviewers for their helpful comments. Correspondence concerning this article should be addressed to Z. Chen, Department of Psychology, University of Canterbury, Private Bag 4800, Christchurch, New Zealand (e-mail: zhe.chen@canterbury.ac.nz).

\section{REFERENCES}

BECK, D. M., \& LAVIE, N. (2005). Look here but ignore what you see: Effects of distractor at fixation. Journal of Experimental Psychology: Human Perception \& Performance, 31, 592-607.

Bennett, P. J., \& Pratt, J. (2001). The spatial distribution of inhibition of return. Psychological Science, 12, 76-80.

Bouma, H. (1973). Visual interference in the parafoveal recognition of initial and final letters of words. Vision Research, 13, 762-782

Boynton, R. M. (1979). Human color vision. New York: Holt, Rinehart and Winston.

Bruce, V. (1988). Recognizing faces. Hillsdale, NJ: Erlbaum.

Carrasco, M., Evert, D. L., Chang, I., \& Katz, S. M. (1995). The eccentricity effect: Target eccentricity affects performance on conjunction searches. Perception \& Psychophysics, 57, 1241-1261.

Carrasco, M., Giordano, A. M., \& McElree, B. (2006). Attention speeds processing across eccentricity: Feature and conjunction searches. Vision Research, 46, 2028-2040.

Carrasco, M., McElree, B., Denisova, K., \& Giordano, A. M. (2003). The speed of visual information processing increases with eccentricity. Nature Neuroscience, 6, 699-700.

Cave, K. R., \& Zimmerman, J. M. (1997). Flexibility in spatial attention before and after practice. Psychological Science, 8, 399-403.

Cepeda, N. J., Cave, K. R., Bichot, N. P., \& Kim, M.-S. (1998). Spatial selection via feature-driven inhibition of distractor locations. Perception \& Psychophysics, 60, 727-746.

Chelazzi, L., Miller, E. K., Duncan, J., \& Desimone, R. (1993). A neural basis for visual search in inferior temporal cortex. Nature, 363, 345-347.

CHEN, Z. (2008). Distractor eccentricity and its effect on selective attention. Experimental Psychology, 55, 82-92.

Connolly, M., \& VAN Essen, D. (1984). The representation of the visual field in parvicellular and magnocellular layers of the lateral geniculate nucleus in the macaque monkey. Journal of Comparative Neurology, 226, 544-564.

DAGEnBACH, D., \& CARR, T. H. (EDS.) (1994). Inhibitory processes in attention, memory, and language. San Diego: Academic Press.

DeSchepper, B. G., \& Treisman, A. M. (1996). Visual memory for novel shapes: Implicit coding without attention. Journal of Experimental Psychology: Learning, Memory, \& Cognition, 22, 27-47.

Desimone, R. (1991). Face-selective cells in the temporal cortex of monkeys. Journal of Cognitive Neuroscience, 3, 1-8.

Dori, H., \& HeNIK, A. (2006). Indications for two attentional gradients in endogenous visual-spatial attention. Visual Cognition, 13, 166-201.

Downing, P. E., \& Pinker, S. (1985). The spatial structure of visual attention. In M. I. Posner \& O. S. M. Martin (Eds.), Attention and 
performance XI: Mechanisms of attention (pp. 171-187). Hillsdale, NJ: Erlbaum

ERIKSEN, B. A., \& ERIKSEN, C. W. (1974). Effects of noise letters upon the identification of a target letter in a nonsearch task. Perception \& Psychophysics, 16, 143-149.

FioRENTINI, A., \& BERARDI, N. (1991). Limits in pattern discrimination: Central and peripheral factors. In J. R. Cronly-Dillon (Ed.), Vision and visual dysfunction (pp. 266-275). Basingstoke, U.K.: Macmillan.

Gauthier, I., Skudlarski, P., Gore, J. C., \& Anderson, A. W. (2000). Expertise for cars and birds recruits brain areas involved in face recognition. Nature Neuroscience, 3, 191-197.

Goolkasian, P. (1999). Retinal location and its effect on the spatial distribution of visual attention. American Journal of Psychology, 112 , 187-214.

Gross, C. G., Rocha-Maranda, C. E., \& Bender, D. B. (1972). Visual properties of neurons in inferotemporal cortex of the macaque. Journal of Neurophysiology, 35, 96-111.

JiAng, Y., \& CHUN, M. M. (2001). Asymmetric object substitution masking. Journal of Experimental Psychology: Human Perception \& Performance, 27, 895-918.

JoNIDES, J. (1981). Voluntary versus automatic control over the mind's eye's movement. In J. B. Long \& A. D. Baddeley (Eds.), Attention and performance IX (pp. 187-203). Hillsdale, NJ: Erlbaum.

Juola, J. F., Koshino, H., \& Warner, C. B. (1995). Tradeoffs between attentional effects of spatial cues and abrupt onsets. Perception \& Psychophysics, 57, 332-342.

Kanwisher, N., McDermott, J., \& Chun, M. M. (1997). The fusiform face area: A module in human extrastriate cortex specialized for face perception. Journal of Neuroscience, 17, 4302-4311.

Kastner, S., \& Pinsk, M. A. (2004). Visual attention as a multilevel selection process. Cognitive, Affective, \& Behavioral Neuroscience, 4, 483-500.

KIm, M.-S., \& CAVE, K. R. (1995). Spatial attention in visual search for features and feature conjunctions. Psychological Science, 6 376-380.

Linnell, K. J., \& Humphreys, G. W. (2004). Attentional selection of a peripheral ring overrules the central attentional bias. Perception $\&$ Psychophysics, 66, 743-751.

Luck, S. J., Chelazzi, L., Hillyard, S. A., \& Desimone, R. (1997). Mechanism of spatial selective attention in areas V1, V2, and V4 of macaque visual cortex. Journal of Neurophysiology, 77, 24-42.

MAck, A., \& Rock, I. (1998). Inattentional blindness. Cambridge, MA: MIT Press.

Milliken, B., Joordens, S., Merikle, P. A., \& Seiffert, A. E. (1998) Selective attention: A reevaluation of the implications of negative priming. Psychological Review, 105, 203-229.

Moran, J., \& Desimone, R. (1985). Selective attention gates visual processing in the extrastriate cortex. Science, 229, 782-784.

Neill, W. T., Valdes, L. A., Terry, K. M., \& Gorfein, D. S. (1992) Persistence of negative priming: II. Evidence for episodic trace retrieval. Journal of Experimental Psychology: Learning, Memory, \& Cognition, 18, 993-1000.

PARK, J., \& KANWISher, N. (1994). Negative priming for spatial locations: Identity mismatching, not distractor inhibition. Journal of Experimental Psychology: Human Perception \& Performance, 20 613-623.

Posner, M. I., \& Cohen, Y. (1984). Components of visual orienting. In H. Bouma \& D. G. Bouwhuis (Eds.), Attention and performance $X$ (pp. 531-556). Hillsdale, NJ: Erlbaum.

Purcell, D., \& Stewart, A. (1986). The face-detection effect. Bulletin of the Psychonomic Society, 24, 118-120.

REED, A. V. (1973). Speed-accuracy trade-off in recognition memory. Science, 181, 574-576.

RoDIEck, R. W. (1973). The vertebrate retina: Principles of structure and function. Oxford: Freeman.

Rovamo, J., \& Virsu, V. (1979). An estimation and application of the human cortical magnification factor. Experimental Brain Research 37, 495-510.

Schneider, W., Eschman, A., \& Zuccolotto, A. (2002). E-Prime user's guide. Pittsburgh: Psychology Software Tools, Inc.

Shulman, G. L., Sheehy, J. B., \& Wilson, J. (1986). Gradients of spatial attention. Acta Psychologica, 61, 167-181.

Smith, A. T., Singh, K. D., \& Greenlee, M. W. (2000). Attentional suppression of activity in the human visual cortex. NeuroReport, 11, 271-277.

Somers, D. C., Dale, A. M., Seiffert, A. E., \& Tootell, R. B. H. (1999). Functional MRI reveals spatially specific attentional modulation in human primary visual cortex. Proceedings of the National Academy of Sciences, 96, 1663-1668.

TIPPER, S. P. (2001). Does negative priming reflect inhibitory mechanisms? A review and integration of conflicting views. Quarterly Journal of Experimental Psychology: Human Experimental Psychology, 54A, 321-343.

TIPPER, S. P., \& DRIVER, J. (1988). Negative priming between pictures and words in a selective attention task: Evidence for semantic processing of ignored stimuli. Memory \& Cognition, 16, 64-70.

Tsal, Y., \& MAKovsKI, T. (2006). The attentional white bear phenomenon: The mandatory allocation of attention to expected distractor location. Journal of Experimental Psychology: Human Perception \& Performance, 32, 351-363.

VIRSU, V., \& Rovamo, J. (1979). Visual resolution, contrast sensitivity, and the cortical magnification factor. Experimental Brain Research, 37, 1-16.

WiCKELGREN, W. A. (1977). Speed-accuracy tradeoff and information processing dynamics. Acta Psychologica, 41, 67-85.

Wolfe, J. M., O'Neill, P., \& Bennett, S. C. (1998). Why are there eccentricity effects in visual search? Visual and attentional hypotheses. Perception \& Psychophysics, 60, 140-156.

\section{NOTES}

1. Without the filler trials, the participants could guess the identity of the target in the incongruent condition (i.e., the target would be the other letter of the target set). Although prior research (Chen, 2008) has shown that using compatible distractors could induce participants not to inhibit distractors, the small proportion of compatible trials we used here made it unlikely that such a strategy would be adopted. Data from these compatible trials were not included in subsequent analyses.

2 . For Experiment $3 \mathrm{~A}$, the mean RTs for the central and peripheral targets were $353 \mathrm{msec}$ (with $8.5 \%$ error) and $358 \mathrm{msec}$ (with $11.3 \%$ error), respectively, for the fast trials $[t(10)=1.73, p=.11$ for RT, and $t(10)=$ $2.56, p<.05$ for accuracy], and they were $479 \mathrm{msec}$ (with $3.8 \%$ error) and $475 \mathrm{msec}$ (with $3.8 \%$ error) for the slow trials $[t(10)=0.61, p=$ .56 for RT, and $t(10)=0.01, p=.99$ for accuracy]. For Experiment 3B, the mean RTs for the central and peripheral targets were $447 \mathrm{msec}$ (with $8.8 \%$ error) and $451 \mathrm{msec}$ (with $8.6 \%$ error) for the fast trials $[t(10)=$ $.67, p=.52$ for RT, and $t(10)=0.08, p=.93$ for accuracy], and they were $607 \mathrm{msec}$ (with $3.6 \%$ error) and $607 \mathrm{msec}$ (with $2.8 \%$ error) for the slow trials $[t(10)=0.08, p=.93$ for RT, and $t(10)=0.97, p=.35$ for accuracy]. It is worth noting that the only significant result was in the accuracy data of Experiment 3A. When RTs were fast, accuracy was lower in the peripheral condition than in the central condition. Note that this result is opposite to what one would predict, if processing is more efficient for a peripheral than for a central stimulus.

3 . We used the neutral distractor on the probe trials to ensure visibility of the probe. The incompatible distractor used in our experiments was either an $\mathrm{X}$ or an $\mathrm{H}$, and both would mask the probe heavily. In contrast, the neutral distractor $\mathrm{L}$ would allow the probe to be more visible, because the probe would not appear at the location occupied by the vertical or horizontal bar of the letter L.

4. The data from the different conditions of the distractor group were collapsed because an ANOVA showed no significant effects of incompatibility $\left[F(1,7)=2.28, M S_{\mathrm{e}}=1.21, p=.17\right]$, distractor location $\left[F(1,7)=1.74, M S_{\mathrm{e}}=3.3, p=.23\right]$, or their interaction $[F(1,7)=.15$, $\left.M S_{\mathrm{e}}=2.5, p=.72\right]$. The mean error rates for the incongruent and neutral trials were $1.95 \%$ and $1.56 \%$ in the central conditions and $2.99 \%$ and $2.22 \%$ in the peripheral conditions, respectively.

5. One may wonder whether this result is consistent with the recent finding of Tsal and Makovski (2006), whose participants perceived a dot at an expected distractor location earlier than a dot at an expected empty location. There is one important difference between our experiment and the study of Tsal and Makovski. In Experiment 4 of the present study, the probe, if present, was shown after the offset of the target display. Participants were required to respond to it before they performed the letter discrimination task. In other words, we measured the allocation of attention immediately after the offset of a distractor 
(in the distractor group) and compared that with the allocation of attention to an empty location (in the no-distractor group). Our results are consistent with the finding of Cepeda et al. (1998), whose participants also showed faster RT when a probe was at an empty location than when it was preceded by a distractor. In contrast, Tsal and Makovski measured the distribution of attention to an expected distractor location versus to an empty location. They randomly mixed probe trials among letter discrimination trials. On some unpredictable trials, the participants were shown two probes, one at an expected distractor location and the other at an expected empty location. Their results show that participants perceived the dot at the expected distractor location to appear earlier than the dot at the expected empty location, suggesting that attention is allocated to a distractor location before the onset of a stimulus display.

6. Two repeated measures ANOVAs with letter (present vs. absent) and dot location (central vs. peripheral) as factors were conducted. For RT, there was no main effect of letter $\left[F(1,7)=0.34, M S_{\mathrm{e}}=424.5, p=.58\right]$ or letter $\times$ location interaction $\left[F(1,7)=0.21, M S_{\mathrm{e}}=147.2, p=.66\right]$. The main effect of location approached significance $[F(1,7)=4.99$, $\left.M S_{\mathrm{e}}=1,842.6, p=.06\right]$, with faster RT for a central $\operatorname{dot}(350 \mathrm{msec})$ than for a peripheral dot (384 msec). For accuracy, no effects were significant $\left[F(1,7)=2.64, M S_{\mathrm{e}}=32.6, p=.15\right.$, for letter; $F(1,7)=2.20, M S_{\mathrm{e}}=$ $39.3, p=.18$, for location; and $F(1,7)=1.13, M S_{\mathrm{e}}=35.1, p=.32$, for letter $\times$ location interaction].

We attributed the faster RT for a central than for a peripheral dot to the distribution of attention in the present experiment. Unlike the letter discrimination tasks described in Experiments $3 \mathrm{~A}$ and $3 \mathrm{~B}$, where the cue occurred at $5^{\circ}$ eccentricity (halfway between the two target locations at $1^{\circ}$ and $9^{\circ}$ eccentricities), the cue in the present experiment always appeared at the location of the target. This means that attention was concentrated within $1^{\circ}$ of fixation on half of the trials, but at the left or right peripheral location on only one fourth of the trials. This could induce participants to pay more attention to the center, resulting in faster RT to a central than to a peripheral dot.

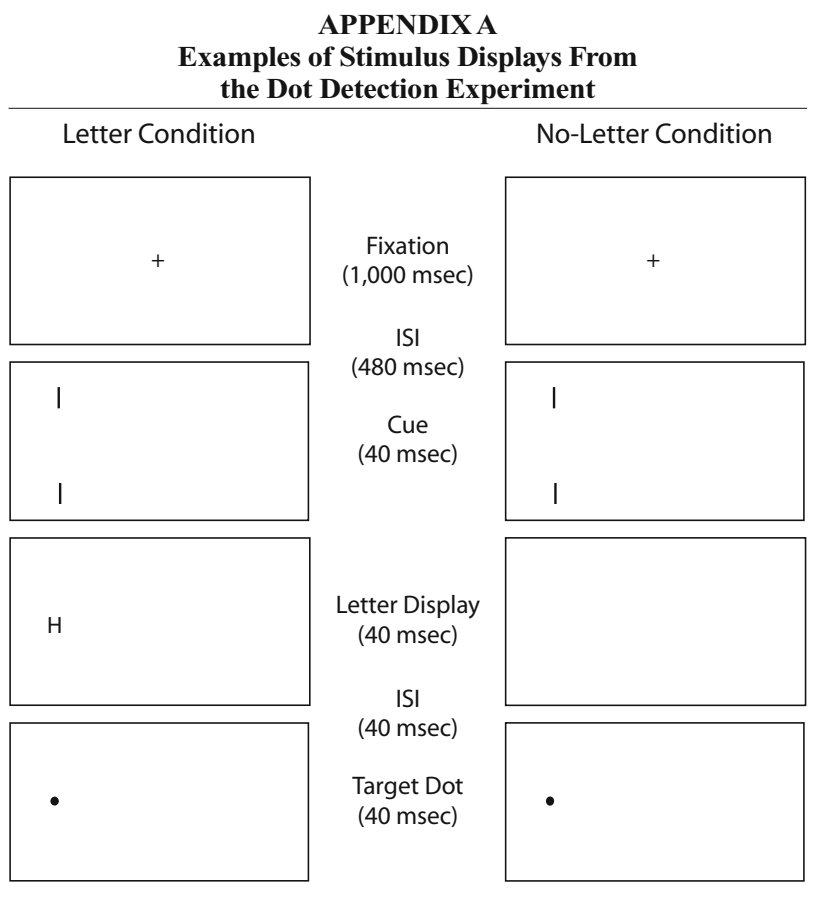

APPENDIX B

Mean Reaction Times (in Milliseconds) and Error Rates (Percentage Incorrect), With Standard Errors, for the Dot Detection Task

\begin{tabular}{|c|c|c|c|c|c|c|c|c|}
\hline & & Letter & nditio & & & -Lette & ondit & ion \\
\hline & $\mathrm{Ce}$ & tral & Peri & heral & $\mathrm{Ce}$ & tral & Perip & heral \\
\hline & $M$ & $S E$ & $M$ & $S E$ & $M$ & $S E$ & $M$ & $S E$ \\
\hline RT & 347 & 19.2 & 382 & 29.3 & 353 & 15.2 & 385 & 23.1 \\
\hline$\%$ Error & 1.4 & 1.0 & 7.0 & 4.0 & 0.4 & 0.3 & 1.5 & 0.6 \\
\hline
\end{tabular}

(Manuscript received April 25, 2007;

revision accepted for publication March 11, 2008.) 